\title{
A non-centralized predictive control strategy for wind farm active power control: a wake-based partitioning approach
}

Owing to wake effects, the power production of each turbine in a wind farm is highly coupled to the operating conditions of the other turbines. Wind farm control strategies must take into account these couplings and produce individual power commands for each turbine. In this case, centralized control approaches might be prone to failures due to the high computational burden and communication dependency. To overcome this problem, this paper proposes a noncentralized scheme based on splitting the wind farm into almost uncoupled sets of turbines by solving a mixed-integer partitioning problem. In each set of turbines, a model predictive control strategy seeks to optimize the distribution of the power set-points among turbines such that the impact of the power losses due to the wake effect is reduced. Then, a supervisory controller coordinates the generation of each group to satisfy the power demanded by the grid operator. The effectiveness of the proposed control scheme in terms of reduction of computational costs and power regulation is confirmed by simulations for a wind farm of 42 turbines.

Keywords: Wind farm control, non-centralized control, partitioning algorithms, model predictive control, wake effect.

\section{Introduction}

With the aim of reducing costs, modern wind farms consist of a large number of turbines. As a result, the power delivered into the electrical grid is close to values provided by conventional power systems. With high penetration of wind energy in the electricity distribution grid, Transmission System Operators (TSOs) demand wind farms to satisfy additional operating conditions and provide functionalities in order to guarantee a reliable network operation [1,2]. This has awaken an increasing interest in academia and industry in wind farm control strategies capable of providing ancillary services, such as frequency and voltage support [3, 4].

On the other hand, a large number of turbines implies a highly complex system to control. Typically, at the wind farm level, a dedicated supervisory computer coordinates the operation of each turbine in order to generate the power demanded by the TSO [5]. This supervisory device needs to gather information from a large number of sensors, compute a large set of commands and send them to each turbine. With larger number of turbines, a centralized control approach requires expensive communication networks, high computation power and reduces the overall system resiliency [6].

An alternative to mitigate the aforementioned issues consists in dividing the wind farm into smaller groups of turbines, denoted as partitions. Each partition is equipped with a controller that communicates only with the turbines in the subset and the supervisor. Thus, communication links and computational costs can be significantly reduced.

\footnotetext{
${ }^{*}$ Corresponding author

Email address: ssiniscalchi@irec. cat (Sara Siniscalchi-Minna)
} 
This idea has been applied to several large-scale systems, e.g., traffic systems, energy systems, smart grids and water systems [7, 8, 9]. The application of partitioning techniques for controlling wind farms in a decentralized manner has been started only recently $[10,11,12]$. One pioneering work in this regard was proposed in [12], in which by exploiting the problem structure a combination of online and offline computations are used to reduce the solving time.

Among modern non-centralized algorithms, alternating direction method of multipliers (ADMM), dual-decomposition and consensus-based control algorithms have attracted more attention for wind farm control [13, 14, 15, 16]. In [13], fast gradient methods via dual-decomposition are used for power regulation and load alleviation, in which most of the computational tasks are shared by local distributed predictive controllers at wind turbine level and reducing the computational cost of the central unit. ADMM was also used in [10] to solve iteratively a clustering-based distributed optimization problem in order to improve yaw misalignment issues of turbines within wind farms and the total power production. Other limited-communication methodologies use consensus algorithms to maximize the power generation and stored kinetic energy $[17,18]$. These consensus algorithms have been successfully used for optimal power-sharing between wind farms and energy storage devices $[16,19]$. Non-centralized control approaches have also been used in wind farms to mitigate negative wake effects in the power production by computing axial induction factors or yaw misalignment $[15,11]$. As these approaches rely on complex wake models and complex non-convex optimization problems, the online implementation might be difficult.

Extending the previous results in [20], the present paper proposes a hierarchical non-centralized model predictive control (MPC) scheme relaying on a virtual partitioning of a large-scale wind farm. The main contributions are:

- Improvement of the partitioning procedure by casting it as a mixed-integer linear optimization problem taking into account the coupling among turbines caused by wakes.

- Design of a three-level MPC scheme aimed to ensure the power regulation imposed by the TSO and seeking to maximize the power reserve available for ancillary service provision. With the wind farm divided into a few almost uncoupled subsets, the wind farm control is stated using a non-centralized scheme in order to reduce computational burden and high information exchange and thus to increasing the system resiliency.

- The evaluation through numerical simulations of the proposed control scheme in a full-scaled wind farm of 42 turbines using SimWindFarm [21].

The remainder of the paper is organized as follows: Section 2 introduces the wind turbine power generation and wake effect. Section 3 presents the optimal partitioning algorithm aimed to divide the wind farm into almost uncoupled smaller parts and the optimal number of turbines per subset. The non-centralized predictive control architecture is designed in Section 4. In Section 5, the proposed partitioning and control schemes are evaluated in a farm of 42 wind turbines using a wind farm simulator including wake interaction among the turbines. Finally, conclusions and future lines are presented in Section 6.

\section{Nomenclature}

$\mathrm{T}_{i} \quad i$-th turbine

$P_{\mathrm{av}, i} \quad$ Available power for $i$-th turbine

$P_{\mathrm{r}, i} \quad$ Reference power for $i$-th turbine

$P_{\mathrm{w}, i} \quad$ Generated power for $i$-th turbine

$P_{\text {av }, i}^{\mathrm{p}} \quad$ Available power for $i$-th subset

$P_{\mathrm{r}, i}^{\mathrm{av}, i} \quad$ Reference power for $i$-th subset

$P_{\mathrm{w}, i}^{\mathrm{r}, i} \quad$ Generated power for $i$-th subset

$P_{\mathrm{w}, \text { tot }} \quad$ Total generated power

$P_{\text {av,tot }} \quad$ Total available power

$P_{\text {dem }} \quad$ Power demanded by TSO

$P_{\text {res }} \quad$ Power reserve $\left(P_{\text {res }}=P_{\text {av,tot }}-P_{\text {w,tot }}\right)$

$v_{\infty} \quad$ Free-stream wind speed

$\phi \quad$ Free-stream wind speed direction $m \quad$ Number of subsets

$\mathcal{K} \quad$ Set of indexes in the subset $\mathcal{K}=\{1, \ldots, m\}$

$\mathcal{P}_{l} \quad l$-th partition

$\mathcal{P}^{*} \quad$ Partition set

$\overline{\mathcal{V}}_{\text {so }} \quad$ Set of source elements

$\overline{\mathcal{V}}_{\text {in }} \quad$ Set of sink elements

$\tau_{i} \quad$ Network resource-feeding indeces

$\mathbb{R} \quad$ Set of real numbers

$\mathbb{N} \quad$ Set of natural numbers

$\mathbf{I}_{n} \quad$ Identity matrix of dimension $n \times n$ 


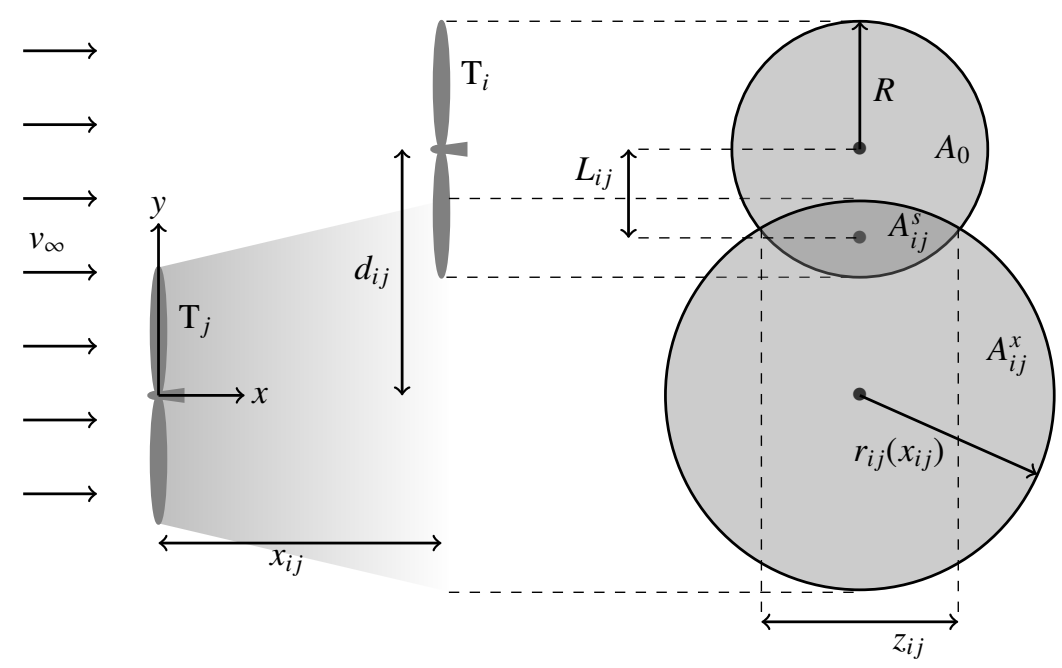

Figure 1: Representation of the wake effect caused by turbine $\mathrm{T}_{j}$ over turbine $\mathrm{T}_{i}$.

\section{Wind Power and Wake Effect}

Considering a wind farm with $n$ turbines, the power generated by the $i$-th turbine $\mathrm{T}_{i}$ is given by

$$
P_{\mathrm{w}, i}=\frac{\rho \pi R^{2}}{2} C_{\mathrm{p}}\left(a_{i}\right) v_{i}^{3}
$$

where $\rho$ is the air density, $R$ is the rotor radius, $v_{i}$ indicates the incoming wind speed and $C_{\mathrm{p}}$ is the power coefficient that depends on the induction factor $a_{i}$, [22].

The wind speed faced and thus the power produced by turbine $\mathrm{T}_{i}$ depend on the free-stream wind speed $v_{\infty}$ and the generation conditions of neighbour turbines. A wind turbine disturbs the air flow producing wakes that expand in the outflow field affecting the speed faced by the downstream turbines.

Several wake models have been proposed in the literature to estimate the wake effect on the wind speed $v_{i}$, the most commonly used being the Park model [23]. This model estimates the effect of multiple wake interactions assuming that wakes expand as a cone-like fashion with circular cross section for a given free-stream wind speed $v_{\infty}$, and the wind speed profile has a top-hat shape in the crosswind direction. Under these assumptions, the wind speed faced by the $i$-th turbine $\mathrm{T}_{i}$ is computed as

$$
v_{i}=v_{\infty}\left(1-2 \sqrt{\sum_{j \in \mathcal{N}_{i}}\left(2\left(1-a_{j}\right) \frac{R}{r_{i j}\left(x_{i j}\right)}\right)^{2} \frac{A_{i j}^{s}\left(x_{i j}\right)}{A_{0, i}}}\right),
$$

where $x_{i j}$ is the distance in the $\mathrm{x}$-direction between turbines $\mathrm{T}_{j}$ and $\mathrm{T}_{i}, r_{i j}\left(x_{i j}\right)=R+z_{0} x_{i j}$ is the radius of the wake generated by turbine $\mathrm{T}_{j}, z_{0}$ the roughness coefficient and $\mathcal{N}_{i}$ is the set of indeces corresponding to the turbines upstream of $\mathrm{T}_{i}$. The symbols $A_{0, i}$ and $A_{i j}^{s}\left(x_{i j}\right)$ denote the rotor area and the shadowed area due to the upstream turbine, respectively (see Figure 1). If the wind turbines have the same radius $R$, then the shadowed area can be computed as

$$
A_{i j}^{s}\left(x_{i j}\right)=r_{i j}\left(x_{i j}\right)^{2} \cos ^{-1}\left(\frac{L_{i j}}{r_{i j}\left(x_{i j}\right)}\right)+R \cos ^{-1}\left(\frac{d_{i j}-L_{i j}}{r_{i j}\left(x_{i j}\right)}\right)+d_{i j} z_{i j},
$$

with $L_{i j}$ the distance between the centres of the wake area $A_{i j}^{x}$ and the shadowed area $A_{i j}^{s}, d_{i j}$ the distance between the centres of the wake area $A_{i j}^{x}$ and the rotor area $A_{0, i}$, and $z_{i j}$ the vertical distance between the intersection points of the previously mentioned areas (see Figure 1).

The wake impact on the wind speed faced by downstream turbines depends on the free-stream wind speed direc- 
tion $\phi$ (defined as the angle between $v_{\infty}$ and the farm layout as indicated in Figure 4) and the turbine geographical disposition within the farm [24]. Hence, the wake effect faced by some turbines can be either partial or total as shown in Figure 1. As stated before, the effect over downstream turbines also depends on the operational conditions of upstream turbines, which are taken into account with the induction factor in (2). Nevertheless, the degree of coupling due to wake effects between turbine $\mathrm{T}_{i}$ and $\mathrm{T}_{j}$ is basically a function of the wind speed direction and wind farm layout (location and distance among turbines).

In large wind farms, couplings among turbines caused by wakes are significant and lead to substantial power losses. Such negative impact can be reduced with suitable control strategies that send power commands to each turbine considering couplings. In this circumstance, centralized control approaches may demand large information sharing between turbines and the central controller. Complex communications and large information exchange result difficult to process over times suitable to satisfy the grid requirements (typically about seconds [25]) and the high communication dependency make the system exposed to failures. For this reason, in this work, a possible solution to mitigate the aforementioned issues is proposed by designing a non-centralized wind farm control strategy. In this approach, turbines are sorted into subsets controlled by independent local controllers, which are local decision makers that use only the portion of information corresponding to the specific subset of turbines.

\section{Wind Farm Partitioning}

As the first step towards optimally designing a non-centralized control strategy, the wind farm is partitioned into several almost uncoupled subsets of turbines. That is, wind turbines are organized in subsets according to the coupling level associated with the wake effect. Among the different approaches proposed for partitioning large-scale systems $[26,8,14]$, here the partitioning approach proposed in [20] is considered and improved in order to provide a more robust partitioning algorithm.

\subsection{Partitioning problem}

With the aim of considering wake effects, the interactions due to the wake propagations are represented as a weighted directed graph $\mathcal{G}=(\mathcal{V}, \mathcal{E})$, where $\mathcal{V}=\{1,2, \ldots, n\}$ is the set of vertices, where each vertex corresponds to a wind turbine and $\mathcal{E}=\{(i, j): i, j \in \mathcal{V}\}$ is the set of edges with weights

$$
\epsilon_{i j}=\left|\frac{R}{r_{i j}\left(x_{i j}\right)}\right| \frac{A_{i j}^{s}\left(x_{i j}\right)}{A_{0}},
$$

if turbine $\mathrm{T}_{i}$ is placed downstream of turbine $\mathrm{T}_{j}$ (i.e., the wind speed faced by the $i$-th turbine is affected by the wake caused by $j$-th turbine); otherwise, $\epsilon_{i j}=0$ [14].

Then, according to the wind farm layout and the predominant free-stream wind speed direction $\phi$, the wind farm can be sorted in $m$ subsets and the number of turbines within each subset can be found by solving the following optimization problem:

$$
\begin{array}{ll}
\underset{\delta_{i l}}{\operatorname{minimize}} & \sum_{q=1}^{3} w_{q} f_{q}\left(\delta_{i l}\right), \\
\text { subject to } & \sum_{i \in \mathcal{V}} \delta_{i l} \geq 1, \quad \forall l \in \mathcal{K}, \\
& \sum_{l \in \mathcal{K}} \delta_{i l}=1, \quad \forall i \in \mathcal{V},
\end{array}
$$

with $\delta_{i l} \in\{0,1\}$ a Boolean decision variable such that $\delta_{i l}=1$ if turbine $\mathrm{T}_{i}$ belongs to subset $l$, with $l \in \mathcal{K}=$ $\{1,2, \ldots, m\}$, and 0 otherwise. The non-empty constraint $(5 b)$ and the exclusive constraint $(5 c)$ ensure that the subsets cannot be empty and turbine $\mathrm{T}_{i}$ can only belong to one subset $l$.

The objective function (5a) consists of three terms weighted by $w_{q}>0(q=1,2,3)$ : 
1. The first term $f_{1}$ in the objective function (5a) is stated as the sum of the edge weights at each partition $l \in \mathcal{K}$, i.e.,

$$
f_{1} \triangleq-\sum_{l \in \mathcal{K}} \sum_{i \in \mathcal{V}} \sum_{j \in \mathcal{V} \backslash\{i\}}\left(\epsilon_{i j}+\epsilon_{j i}\right) \delta_{i l} \delta_{j l}
$$

Depending on farm layout and the $v_{\infty}$ direction, only a number of downstream turbines is affected by the wake caused by an upstream turbine. The aim of this term is to consider the wake interactions among the turbines, such that the coupling levels among turbines in the same partition is maximized in order to ensure that turbines coupled by the same wake belong to the same subset.

2. The second term is added to (5a) in order to minimize the distance $d_{i j}$ between turbines belonging to the same subsets, i.e.,

$$
f_{2} \triangleq \sum_{l \in \mathcal{K}} \sum_{i \in \mathcal{V}} \sum_{j \in \mathcal{V} \backslash\{i\}} d_{i j} \delta_{i l} \delta_{j l} .
$$

There might be cases where there is no coupling among turbines, i.e., $\sum_{i \in \mathcal{V}} \sum_{j \in \mathcal{Y} \backslash\{i\}}\left(\epsilon_{i j}+\epsilon_{j i}\right)=0$, e.g., when wind turbines are located in a row (or a column) and the freestream wind direction is such that the wakes generated by the upstream turbines do not affect any other turbine. In such a case, to guarantee a unique solution, turbines can be arranged according to their proximity.

3. Finally, in order to balance the number of turbines in each subset, an extra term is added to minimize the difference between the number of turbines among all subsets, i.e.,

$$
f_{3} \triangleq \sum_{l=1}^{m-1} \sum_{l^{\prime}=l+1}^{m}\left|\sum_{i \in \mathcal{V}} \delta_{i l}-\sum_{j \in \mathcal{V}} \delta_{j l^{\prime}}\right|
$$

Avoiding significant differences between the number of nodes in the subsets will balance the computational burden of finding the optimal solution for each subset.

Setting the weights $w_{q}$, the aforementioned objectives are hierarchically prioritized to find the optimal partition $\mathcal{P}^{*}=\left\{\mathcal{P}_{1}, \ldots, \mathcal{P}_{m}\right\}$

The optimization problem (5) is nonlinear; however it can be recast as a mixed-integer linear programming problem as follows. The procedure to transform products of logical variables, in terms of linear inequalities was presented in [27], which however requires the introduction of auxiliary Boolean variable $\delta_{i j l}$ such that $\delta_{i j l} \triangleq \delta_{i l} \delta_{j l}$. Notice that $\delta_{i j l}=1$ if and only if $\delta_{i l}=1$ and $\delta_{j l}=1$, and therefore

$$
\delta_{i j l}=\left\{\begin{array}{l}
-\delta_{i l}+\delta_{i j l} \leq 0, \\
-\delta_{j l}+\delta_{i j l} \leq 0, \\
\delta_{i l}+\delta_{j l}-\delta_{i j l} \leq 1 .
\end{array}\right.
$$

Let us also define a dummy variable $\varrho_{l l^{\prime}} \in \mathbb{R}$ such that $\varrho_{l l^{\prime}} \geq\left|\sum_{i \in \mathcal{V}} \delta_{i l}-\sum_{i \in \mathcal{V}} \delta_{j l^{\prime}}\right|$, then (8) can be replaced by

$$
f_{3}=\sum_{l=1}^{m-1} \sum_{l^{\prime}=l+1}^{m} \varrho_{l l^{\prime}}
$$


Therefore, the optimization problem (5) becomes

$$
\begin{array}{cl}
\underset{\delta_{i j l}, \varrho_{l l^{\prime}} \cdot}{\operatorname{minimize}} & \sum_{q=1}^{3} w_{q} f_{q}\left(\delta_{i j l}, \varrho_{l l^{\prime}}\right), \\
\text { subject to } & \sum_{i \in \mathcal{V}} \sum_{j \in \mathcal{V} \backslash\{i\}} \delta_{i j l} \geq 1, \quad \forall l \in \mathcal{K}, \\
\sum_{l \in \mathcal{K}} \delta_{i j l} & =1, \quad \forall i, j \in \mathcal{V}, \\
-\delta_{i l}+\delta_{i j l} \leq 0, \quad \forall i, j \in \mathcal{V}, \forall l \in \mathcal{K}, \\
-\delta_{j l}+\delta_{i j l} \leq 0, \quad \forall i, j \in \mathcal{V}, \forall l \in \mathcal{K}, \\
\delta_{i l}+\delta_{j l}-\delta_{i j l} \leq 1, \quad \forall i, j \in \mathcal{V}, \forall l \in \mathcal{K}, \\
\sum_{i \in \mathcal{V}} \delta_{i l}-\sum_{j \in \mathcal{V}} \delta_{j l^{\prime}} \leq \varrho_{l l^{\prime}}, \quad 1 \leq l \leq m-1, \\
\sum_{i \in \mathcal{V}} \delta_{i l}-\sum_{j \in \mathcal{V}} \delta_{j l^{\prime}} \geq-\varrho_{l l^{\prime}}, \quad 1 \leq l \leq m-1,
\end{array}
$$

where $l^{\prime}=l+1$ and $f_{q}(q=1,2,3)$ are given in (6), (7) and (9). As a consequence of using the auxiliary Boolean variable $\delta_{i j l}$, constraints (10d)-(10f) must be added to the original problem (5), [27] and the original no-empty and exclusive constraints in (5b) and (5c) must be rewritten as (10b) and (10c), respectively. Additional constraints (10g) and (10h) are needed to be able to use the dummy variable $\varrho_{l l^{\prime}}$ and hence using (9) instead of (8).

\subsection{Number of subsets}

In order to solve the $m$-partitioning problem (10), it is necessary to provide the number of subsets $m$. A detailed strategy to determine this number for a drinking water network is proposed in [26]. In the current work, a similar approach is presented assuming that the air flow within a wind farm can be modeled as a simplified flow-based distribution network. Many engineering systems have been modeled as flow-based distribution systems [9, 26], which consist of several elements of diverse nature, e.g., storage, actuator, joint, sink, source and flow. Unlike other energy sources, wind cannot be stored, and hence the wind flow in a farm can be obtained identifying only the following elements:

1. Source: element generating the resource. It is equivalent to the turbine facing the free-stream wind condition $v_{\infty}$ and generating the wake in the outflow field. The set of these elements is denoted by $\overline{\mathcal{V}}_{\text {so }}$.

2. Actuator: element that receives and provides the resources. The set of actuator elements is denoted by $\overline{\mathcal{V}}_{\text {ac }}$ and corresponds to the set of turbines increasing the wake effect generated by the upstream turbines $j \in \overline{\mathcal{V}}_{\text {so }}$ proportionally to the operational conditions and, in turn, affected by the wakes generated by the upstream turbines.

3. Sink: element that receives the resource from either the source and/or the actuator. It is equivalent to the turbine only receiving wakes, e.g., the most downstream turbine. The set of sinks is denoted by $\overline{\mathcal{V}}_{\mathrm{si}}$.

4. Link: directed link $(i, j)$ allowing resource flow from an element $i$ to $j$. For a wind farm, this link corresponds to the wake generated by the turbine $i \in \overline{\mathcal{V}}_{\text {so }} \cup \overline{\mathcal{V}}_{\text {ac }}$ and moving through a turbine $j \in \overline{\mathcal{V}}_{\text {ac }} \cup \overline{\mathcal{V}}_{\text {si }}$. The set of link elements is denoted by $\overline{\mathcal{E}} \subset\{(i, j): i, j \in \overline{\mathcal{V}}\}$, with $\overline{\mathcal{V}}=\overline{\mathcal{V}}_{\text {so }} \cup \overline{\mathcal{V}}_{\text {si }} \cup \overline{\mathcal{V}}_{\text {ac }}$.

Therefore, the flow-based distribution system can be identified as a directed graph $\overline{\mathcal{G}}=(\overline{\mathcal{V}}, \overline{\mathcal{E}})$ where each element $i \in \overline{\mathcal{V}}$ has a direct relationship with the turbines in the graph $\mathcal{G}$. The introduced elements for a flow-based distribution system and the representation of the system by a directed graph allow finding the number of subsets as proposed in [26], where three further indicators are defined: 


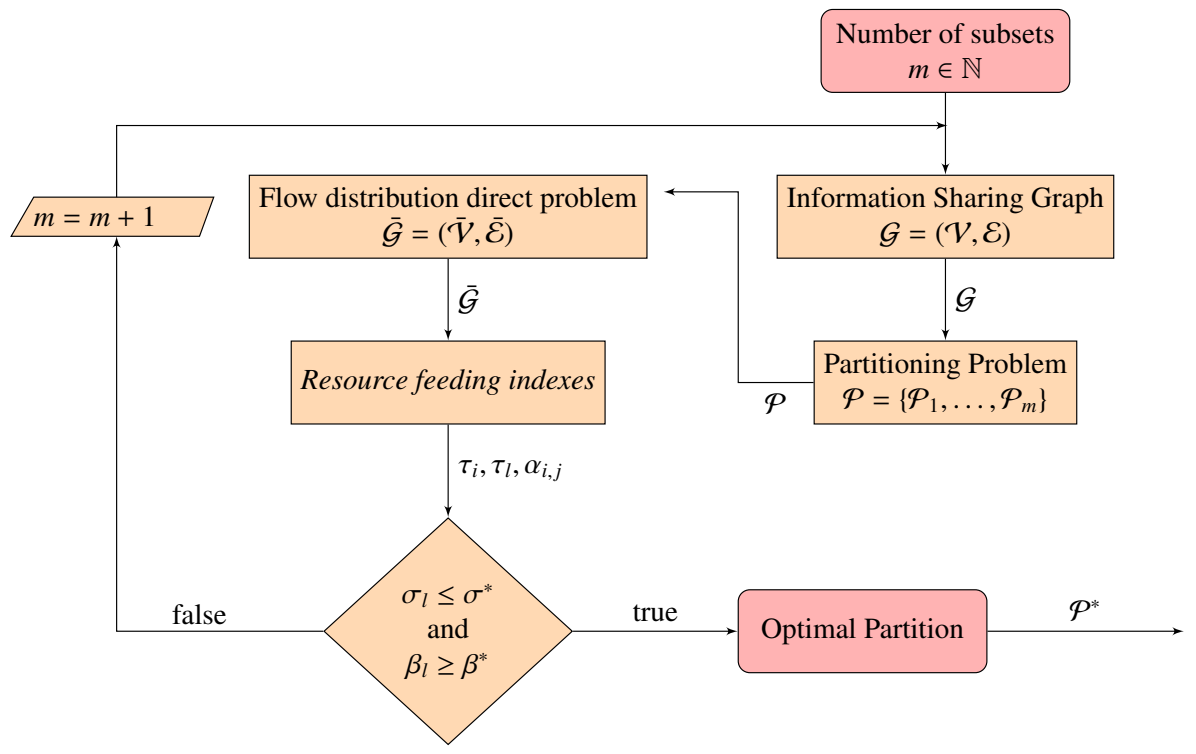

Figure 2: Optimal partitioning algorithm flowchart.

1. Network resource-feeding index, denoted by $\tau_{i}$ with $i \in \overline{\mathcal{V}} \backslash \overline{\mathcal{V}}_{\mathrm{so}}$, gives information about the number of sources or actuator elements that provide the wakes for the element $i \in \overline{\mathcal{V}} \backslash \overline{\mathcal{V}}_{\text {so. }}$. Assuming the graph $\overline{\mathcal{G}}$ is split into $m$ subsets, it is possible to identify the subsets of sinks $\overline{\mathcal{V}}_{\mathrm{si}, l}$ and sources $\overline{\mathcal{V}}_{\mathrm{so}, l}$ for each partition $l \in \overline{\mathcal{K}}, \overline{\mathcal{K}}=\{1, \ldots, m\}$, while the maximum resource-feeding index per partition is defined by $\tau_{l}^{*}=\max _{i \in \overline{\mathcal{V}}_{\mathrm{si}, l}} \tau_{i}$.

2. Sink co-relation index: The proportion of sinks in each subset $l \in \overline{\mathcal{K}}$ with respect the total number of sinks in the flow-based graph $\overline{\mathcal{G}}$, i.e., $\sigma_{l}=\sum_{i \in \overline{\mathcal{V}}_{\mathrm{sil},}} \tau_{i} / \sum_{i \in \overline{\mathcal{V}}_{\mathrm{si}}} \tau_{i}$.

3. Resource-feeding co-relation index: The availability of sources in the subset $l \in \mathcal{K}$ feeding the sink element $i \in \overline{\mathcal{V}}_{\mathrm{si}, l}$ for which $\tau_{i}=\tau_{l}^{*}$. It is assessed with respect to the total number of sources $j \in \overline{\mathcal{V}}_{\text {so }}$ feeding the element $i$, i.e., $\beta_{l}=\sum_{j \in \overline{\mathcal{V}}_{\mathrm{so}, l}} \alpha_{i j} / \sum_{j \in \overline{\mathcal{V}}_{\mathrm{so}}} \alpha_{i j}$, where $\alpha_{i j}=1$ if the flow element $\epsilon_{i j}=1$ with $\epsilon_{i j} \in \overline{\mathcal{E}}$, and 0 otherwise.

The number of subsets is assessed by setting both a desired maximum sink co-relation index and a minimum resourcefeeding co-relation index, hence the subsets should satisfy $\sigma_{l} \leq \sigma^{*}$ and $\beta_{l} \geq \beta^{*}$. The parameter $\sigma^{*}$ is set to ensure that the number of sink elements is balanced among the subsets such that there is no subset without sinks. Furthermore, identifying for each subset the maximum resource-feeding index $\tau_{l}^{*}$ and their respective sources, it is desired that each subset includes a minimum number of these sources indicated by $\beta^{*}$.

\subsection{Algorithm and implementation aspects}

Figure 2 summarizes the partitioning approach proposed in this paper. Assuming an initial number of subsets $m$, the partitioning problem for the information sharing graph $\mathcal{G}$ is first solved and the optimal partition set $\mathcal{P}^{*}$ is found. Then, the analogy with the flow-based distribution graph $\overline{\mathcal{G}}$ is used to check whether or not the criteria for the proper number of subsets are fulfilled. An iterative loop is repeated increasing the initial number of subsets $m=m+1$ until the aforementioned criteria for defining the number of subsets are satisfied.

The partition obtained with the previous procedure $\mathcal{P}^{*}$ depends on the distances $x_{i j}$ and the wind turbine arrangement within the farms (i.e. the set of downstream and upstream turbines), which in turn depends on the free-stream wind speed direction $\phi$. Furthermore, the computational burden to solve the proposed partitioning problem for largescale wind farms can be high and inconsistent with the time scale related to the variation of wind direction and the sampling time used into the wind farm controller. Nevertheless, the subsets can be determined offline and one can keep a look-up table to update the subsets whenever the predominant free-stream wind speed direction changes. As the wind speed direction is sensitive to turbulence and other atmospheric and geographical conditions such as eventual obstacles surroundings, the predominant wind speed direction can be used to select the corresponding subset. 


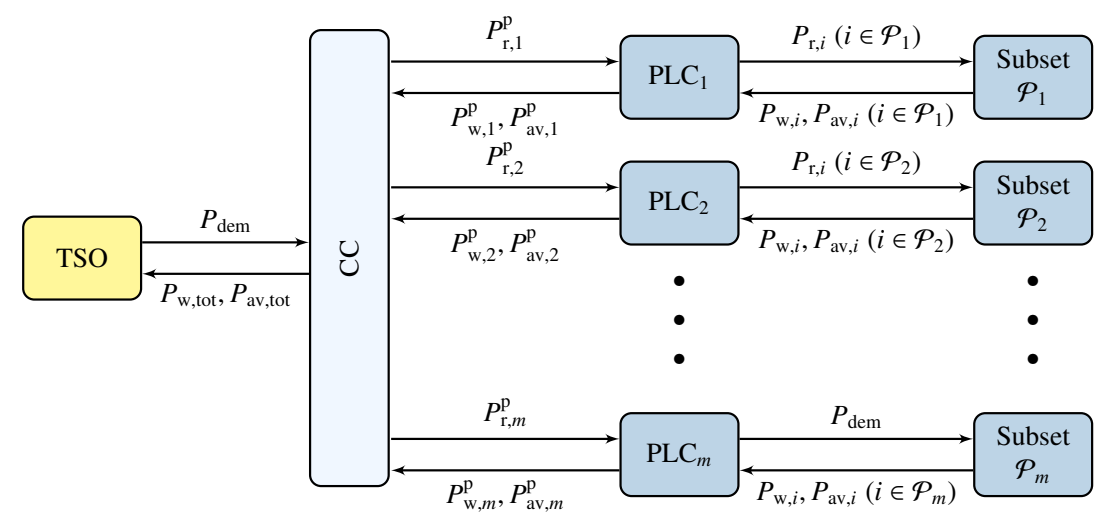

Figure 3: Scheme of the proposed wind farm non-centralized control strategy for a given $\phi$ and the corresponding partition set $\mathcal{P}^{*}$ with $m$ elements.

\section{Wind Farm Control Strategy}

Once the system is partitioned as indicated in the previous section for a set of predominant free-stream wind speed directions

$$
\Phi=\left\{\phi_{1}, \ldots, \phi_{w}\right\}
$$

there is a set $\mathcal{P}^{*}$ of optimal partition sets for each angle $\phi \in \Phi$. Then, for a given direction $\phi$ and the corresponding partition set

$$
\mathcal{P}^{*}=\left\{\mathcal{P}_{1}, \ldots, \mathcal{P}_{m}\right\},
$$

the proposed non-centralized hierarchical control approach is structured as indicated in Figure 3, where $\mathcal{P}_{l}$ is a set of $n_{l}$ indexes corresponding to the wind turbines in the subset $l \in \mathcal{K}=\{1, \ldots, m\}$. For the sake of clarity, Figure 3 illustrates only the signal interactions in the control algorithm and does not include the electrical interconnections.

At the highest level, the Central Controller (CC) collects information regarding the generated power $P_{\mathrm{w}, l}^{\mathrm{p}}$ and the available power $P_{\mathrm{av}, l}^{\mathrm{p}}$ in each subset and then sends the corresponding commands $P_{\mathrm{r}, l}^{\mathrm{p}}$. This controller aims to ensure that the total power delivered at the Point of Common Coupling (PCC) by the wind farm $P_{\mathrm{w}, \text { tot }}$ matches the TSO's power demand $P_{\mathrm{dem}}$. In a lower level, the Partition Level Controllers (PLC) use the measure of the generated power $P_{\mathrm{w}, i}$ and the available power $P_{\mathrm{av}, i}$ at each turbine in the corresponding subset to impose a reference $P_{\mathrm{r}, i}$ to each turbine. Finally, at the lowest level, the wind turbine control guarantees that the generated power satisfies the set-point $P_{\mathrm{r}, i}$.

\subsection{Wind turbine controller}

Each wind turbine is equipped with a power controller that allows working in derated mode if necessary [22, 21]. Thus, the power generated by the $i$-th wind turbine is given by

$$
\dot{P}_{\mathrm{w}, i}=-\frac{1}{\mu}\left(P_{\mathrm{w}, i}-\min \left(P_{\mathrm{av}, i}, P_{\mathrm{r}, i}\right)\right)
$$

where $\mu$ is a time constant, $P_{\mathrm{r}, i}$ is the reference sent by the PLC, and

$$
P_{\mathrm{av}, i}=\min \left(\frac{\rho \pi R^{2}}{2} C_{\mathrm{p}, \max } v_{i}^{3}, P_{\text {rated }}\right)
$$

is the wind turbine available power, with $C_{\mathrm{p}, \max }=\max _{a_{i}} C_{\mathrm{p}}\left(a_{i}\right)$ and $P_{\text {rated }}$ the wind turbine rated power.

\subsection{Partition level controllers (PLCs)}

The PLCs aim to ensure the total power generated in each partition matches the power demanded by the CC. In addition, these controllers seek to distribute the power contribution of each turbine in order to maximize the total available power, which in turn maximizes the power reserve of the entire farm available for ancillary services. The power reserve is defined as $P_{\text {res }}=P_{\text {av,tot }}-P_{\mathrm{w}, \text { tot }}$. 
For a given partition $\mathcal{P}_{l}$, with $l \in \mathcal{K}$ and $n_{l}$ turbines, the corresponding PLC relies on an MPC strategy based on the following optimization problem:

$$
\begin{array}{cc}
\underset{\mathbf{u}_{l}(k)}{\operatorname{minimize}} & \sum_{q=1}^{3} \sum_{k=1}^{H_{\mathrm{p}}-1} \gamma_{q} J_{q}\left(\mathbf{x}_{l}(k), \mathbf{u}_{l}(k)\right) \\
\text { subject to } & \mathbf{x}_{l}(k+j+1 \mid k)=\mathbf{A}_{d, l} \mathbf{x}_{l}(k+j \mid k)+\mathbf{B}_{d, l} \mathbf{u}_{l}(k+j \mid k), \\
P_{\min } & \leq u_{l, i}(k+j) \leq P_{\mathrm{av}, i}, \quad \forall i \in \mathcal{P}_{l}
\end{array}
$$

where $\gamma_{q}>0$ are prioritization weights such that $\sum_{q=1}^{3} \gamma_{q}=1, \mathbf{x}_{l} \in \mathbb{R}^{n_{l}}$ is the state vector and $\mathbf{u}_{l} \in \mathbb{R}^{n_{l}}$ is the vector of manipulated variables, with elements $x_{l, i}=P_{\mathrm{w}, i}$ and $u_{l, i}=P_{\mathrm{r}, i}\left(i \in \mathcal{P}_{l}\right)$, respectively. The time indices $k \in \mathbb{N}$, $j \in\left\{0, \ldots, H_{\mathrm{p}}-1\right\}$ and the prediction horizon $H_{\mathrm{p}}$ are defined such that $\mathbf{x}_{l}(k+j \mid k)$ denotes the vector of measured generated power at the instant $k$ corresponding to the control input $\mathbf{u}_{l}(k+j \mid k)$.

The solution of problem (13) is the optimal control input $\hat{\mathbf{u}}_{l} \triangleq \hat{\mathbf{u}}_{l}(k \mid k)$ corresponding to the set-points for each the turbine $i \in \mathcal{P}_{l}$. Notice that the first constraint (13b) corresponds to the discretized version of (11), used to predict the power response of the wind turbines in the partition $\mathcal{P}_{l}$, where $\mathbf{A}_{d, l}$ and $\mathbf{B}_{d, l}$ are the discretized versions of the matrices

$$
\mathbf{A}_{l}=-(1 / \mu) \mathbf{I}_{n_{l}}, \quad \mathbf{B}_{l}=(1 / \mu) \mathbf{I}_{n_{l}} .
$$

Finally, in the last constraint (13b), $P_{\min }$ denotes the minimum power used as a lower bound to avoid solutions implying the shutting-down of some turbines.

The cost function (13a) covers three objectives:

1. Minimizing the tracking error, i.e., $J_{1}\left(\mathbf{x}_{l}(k), P_{\mathrm{r}, l}^{\mathrm{p}}(k)\right) \triangleq\left\|P_{\mathrm{r}, l}^{\mathrm{p}}(k)-\sum_{i=1}^{n_{l}} x_{l, i}(k)\right\|_{2}$, where $P_{\mathrm{r}, l}^{\mathrm{p}}$ is the set-point imposed by the $\mathrm{CC}$.

2. Maximizing the available power, i.e., $J_{2}\left(\mathbf{u}_{l}(k)\right) \triangleq\left\|\mathbf{R} \mathbf{u}_{l}(k)\right\|_{2}$, where the elements of the matrix $\mathbf{R}$ are defined as

$$
[\mathbf{R}]_{i j}= \begin{cases}\left(\tau_{i}+\kappa\right)^{-\lambda}, & \text { if } i=j, \lambda=\max \left(0,\left(P_{\mathrm{av}, l}^{\mathrm{p}}-P_{\mathrm{r}, l}^{\mathrm{p}}\right) / P_{\mathrm{av}, l}^{\mathrm{p}}\right), \\ 0, & \text { if } i \neq j .\end{cases}
$$

Here $\tau_{i}$ is the network resource-feeding index introduced in Section $3, P_{\mathrm{av}, l}^{\mathrm{p}}$ is the total available power in the subset $\mathcal{P}_{l}$ and $K>0$ is a small constant to avoid singularity when the turbines are not affected by wakes.

When $P_{\mathrm{r}, l}^{\mathrm{p}}$ is lower than the total available power, $J_{1}=0$ can be achieved with different power contributions from each turbine. This degree-of-freedom can be used to maximize the available power and thus the power reserve. Inspired by the backward scheme presented in [28], here a simpler approach is proposed based on penalizing the contributions of the most upstream turbines. The idea consists in reducing the contribution of the upstream turbines to reduce the wind speed deficits faced by the downstream turbine.

As the power demand $P_{\mathrm{r}, l}^{\mathrm{p}}$ is close to the available power, the backward distribution may not be effective. In order to mitigate this issue, the exponent $\lambda(0 \leq \lambda \leq 1)$, defined as the ratio between the power reserve and the available power, is reduced. Thus, in circumstance of high power demands, all turbines contribute with the same power, whereas, for higher derating operations, the backward distribution is used.

3. Limiting fast variations of the control inputs to smooth the operation and avoid possible damage on the turbine, $J_{3}\left(\mathbf{u}_{l}(k)\right) \triangleq\left\|\mathbf{u}_{l}(k)-\mathbf{u}_{l}(k-1)\right\|_{2}$.

\subsection{Central Controller $(C C)$}

The aim of the $\mathrm{CC}$ is to ensure the entire wind farm delivers the power $P_{\mathrm{dem}}$ required by the TSO. To this end, the CC receives, from each PLC, information about the total generated power $P_{\mathrm{w}, l}^{\mathrm{p}}$ and the total available power $P_{\mathrm{av}, l}^{\mathrm{p}}$ corresponding to the partition, and then produces a set of power references for each subset $P_{\mathrm{r}, l}^{\mathrm{p}}$.

As a consequence of the partitioning procedure, all turbine subsets can be considered uncoupled. Moreover, as only the total power is relevant to this analysis and in order to keep the controller simple, the dynamic response of 
each partition can be described by a first-order system representing an aggregated wind turbine as follows

$$
\dot{P}_{\mathrm{w}, l}^{\mathrm{p}}=-\frac{1}{\mu_{l}}\left(P_{\mathrm{w}, l}^{\mathrm{p}}-P_{\mathrm{r}, l}^{\mathrm{p}}\right) .
$$

where $\mu_{l}$ is a time constant that depends on the number of turbines in $\mathcal{P}_{l}$ and the PLC.

The CC relies on an MPC strategy based on the following optimization problem:

$$
\begin{aligned}
& \underset{\mathbf{u}_{\mathrm{p}}(k)}{\operatorname{minimize}} \sum_{k=1}^{H_{\mathrm{p}}-1}\left\|\mathbf{Q} \mathbf{x}_{\mathrm{p}}(k)\right\|_{2}+\left\|\mathbf{S}\left(\mathbf{u}_{\mathrm{p}}(k)-\mathbf{u}_{\mathrm{p}}(k-1)\right)\right\|_{2} \\
& \text { subject to } \quad \mathbf{x}_{\mathrm{p}}(k+j+1 \mid k)=\mathbf{E}_{d} \mathbf{x}_{\mathrm{p}}(k+j \mid k)+\mathbf{F}_{d} \mathbf{u}_{\mathrm{p}}(k+j \mid k)+\mathbf{G}_{d} P_{\mathrm{dem}}(k+j \mid k), \\
& \mathbf{P}_{\min }^{\mathrm{p}} \leq \mathbf{u}_{\mathrm{p}}(k+j) \leq \mathbf{P}_{\mathrm{av}}^{\mathrm{p}},
\end{aligned}
$$

with $k \in \mathbb{N}, j \in\left\{0, \ldots, H_{\mathrm{p}}-1\right\}$, and $H_{\mathrm{p}}$ the prediction horizon. As for the PLC, (14b) corresponds to the discrete version of the following approximated dynamic model of the entire wind farm:

$$
\dot{\mathbf{x}}_{\mathrm{p}}(t)=\mathbf{E} \mathbf{x}_{\mathrm{p}}(t)+\mathbf{F} \mathbf{u}_{\mathrm{p}}(t)+\mathbf{G} P_{\mathrm{dem}}(t)
$$

where

$$
\begin{aligned}
& \mathbf{x}_{\mathrm{p}}=\left[\begin{array}{llll}
P_{\mathrm{w}, 1}^{\mathrm{p}} & \ldots & P_{\mathrm{w}, m}^{\mathrm{p}}, & \xi
\end{array}\right]^{T}, \\
& \xi=\left(P_{\mathrm{dem}}-\sum_{i=1}^{m} P_{\mathrm{w}, i}^{\mathrm{p}}\right), \\
& \mathbf{u}_{\mathrm{p}}=\left[\begin{array}{lll}
P_{\mathrm{r}, 1}^{\mathrm{p}} & \ldots & P_{\mathrm{r}, m}^{\mathrm{p}}
\end{array}\right]^{T} \text {, } \\
& \mathbf{E}=\left[\begin{array}{cccc}
-1 / \mu_{1} & 0 & \ldots & 0 \\
0 & \ddots & \vdots & \vdots \\
\vdots & & -1 / \mu_{m} & \vdots \\
-1 & \ldots & -1 & 0
\end{array}\right] \text {, } \\
& \mathbf{F}=\left[\begin{array}{ccc}
1 / \mu_{1} & 0 & \ldots \\
0 & \ddots & \vdots \\
\vdots & & 1 / \mu_{m} \\
0 & \ldots & 0
\end{array}\right] \\
& \mathbf{G}=\left[\begin{array}{c}
0 \\
\vdots \\
0 \\
1
\end{array}\right]
\end{aligned}
$$

Constraint (14c) ensures the power references remain within the operating limits given by the minimum power

$$
\mathbf{P}_{\min }^{\mathrm{p}}=\left[\begin{array}{lll}
n_{1} P_{\min } & \ldots & n_{m} P_{\min }
\end{array}\right]^{T}
$$

to avoid shutting-down partitions and the available power defined as

$$
\mathbf{P}_{\mathrm{av}}^{\mathrm{p}}=\left[\begin{array}{lll}
P_{\mathrm{av}, 1}^{\mathrm{p}} & \cdots & P_{\mathrm{av}, m}^{\mathrm{p}}
\end{array}\right]^{T},
$$

where $P_{\mathrm{av}, l}^{\mathrm{p}}=\sum_{i \in \mathcal{P}_{l}} P_{\mathrm{av}, i}$.

In the cost function (14a) is the sum of two objectives, the first is included to minimize the error in the tracking of the power demand. Therefore, the matrix $\mathbf{Q}=\operatorname{diag}\left(0, \ldots, 0, Q_{\xi}\right)$ penalizes only the integral of the tracking error. The second objective is related to the smooth operation and, the matrix $\mathbf{S}$ penalizes the rate of variation of the power references.

\section{Case Study}

The proposed partitioning approach and control strategy were tested for a wind farm of 210 MW rated power with 42 benchmark NREL-5MW wind turbines [29] spaced $630 \mathrm{~m}$ (i.e. 5 rotor diameters) and placed as shown in Figure 4. The wind field and wake effect have been simulated for the free-stream wind speed of $v_{\infty}=11 \mathrm{~m} / \mathrm{s}$ using 
Table 1: Sinks, resource feeding indexes and sources in the directed graph $\overline{\mathcal{G}}$ and their connection with the turbines in the information sharing graph G.

\begin{tabular}{lll}
\hline $\mathrm{T}_{i}$ Sink, $i \in \mathcal{V}_{\mathrm{si}}$ & $\tau_{i}, i \in \mathcal{V}_{\mathrm{si}}$ & $\mathrm{T}_{j}$ Source, $j \in \mathcal{V}_{\text {so }}$ \\
\hline $\mathrm{T}_{37}$ & 1 & $\mathrm{~T}_{29}$ \\
$\mathrm{~T}_{38}, \mathrm{~T}_{39}, \mathrm{~T}_{10}, \mathrm{~T}_{13}, \mathrm{~T}_{14}$ & 2 & $\mathrm{~T}_{(29,30)}, \mathrm{T}_{(22,30)}, \mathrm{T}_{(15,22)}, \mathrm{T}_{(4,5)}, \mathrm{T}_{(6,7)}$ \\
$\mathrm{T}_{41}, \mathrm{~T}_{42}, \mathrm{~T}_{35}, \mathrm{~T}_{27}, \mathrm{~T}_{28}, \mathrm{~T}_{21}$ & 3 & $\mathrm{~T}_{(15,16,22)}, \mathrm{T}_{(8,15,16)}, \mathrm{T}_{(1,8,16)}, \mathrm{T}_{(1,2,8)}, \mathrm{T}_{(1,2,3)}, \mathrm{T}_{(2,3,4)}$ \\
\hline
\end{tabular}

SimWindFarm [21], a MATLAB/Simulink toolbox for wind farm simulation and control. The MPC controllers were implemented with YALMIP [30] and CPLEX.

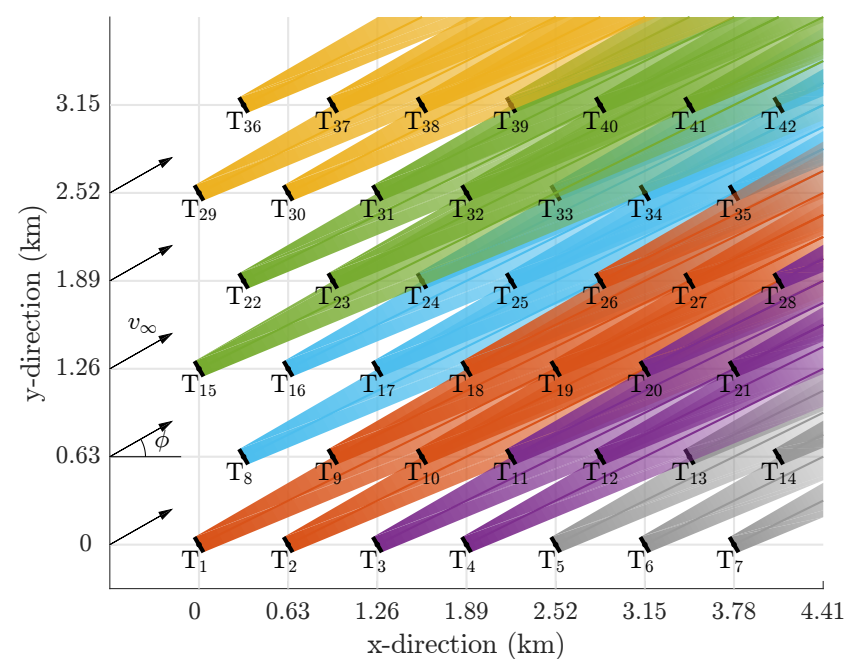

Figure 4: Wind farm layout and optimal partitioning for a wind speed direction of $\phi=30^{\circ}$ : light blue $\mathcal{P}_{1}$, red $\mathcal{P}_{2}$, green $\mathcal{P}_{3}$, yellow $\mathcal{P}_{4}$, gray $\mathcal{P}_{5}$, and purple $\mathcal{P}_{6}$.

\section{Wind farm partitioning}

As mentioned in Section 3, the proposed partitioning approach depends on the predominant wind speed direction $\phi$ and is time-consuming for wind farm layout as the analyzed in this section. Therefore, the partitions were computed offline for the set of angles

$$
\Phi=\left\{\phi=(30 \cdot i)^{\circ}, \quad i=0,1, \ldots, 11\right\} .
$$

A justification of this selection can be found in Section 5.2. The partition obtained using the procedure in Section 3 for a wind speed direction of $\phi=30^{\circ}$ is illustrated in Figure 4 . The flow-based distribution graph $\overline{\mathcal{G}}=(\overline{\mathcal{V}}, \overline{\mathcal{E}})$ is obtained by relating each turbine in the information sharing graph $\mathcal{G}$ to an element of the flow based distribution system. The network resource feeding indices $\tau_{\mathrm{i}}$ for each sink $i \in \mathcal{V}_{\mathrm{si}}$ and the respective sources $j \in \mathcal{V}_{\text {so }}$ for the investigated farm layout are given in Table 1 .

The appropriate number of subsets in the partition was determined based on two conditions: 1) the maximum sink co-relation index has been set as $\sigma^{*}=0.3$, i.e., on each subset the number of sink elements is lower than the $30 \%$ of the total amount of sink elements. The first conditions ensures that the number of sinks is almost balanced among the subsets. 2) The minimum resource feeding co-relation index $\beta^{*}$ per subset was set at 0.5 , i.e., at least half of the source elements feeding the most affected sink in the subset is included in the same subset. These conditions have been defined since they provided the number of subsets that ensures a suitable trade-off between power generation performance and computational burden for solving the proposed optimal control, as it will be presented in the dedicated case study in paragraph Section 5.3. Notice that further conditions may be added to determine the initial 
Table 2: Time constants $\mu_{l}$ and the number of turbines in each subset $\mathcal{P}_{l}$ for different wind speed directions.

\begin{tabular}{|c|c|c|c|c|c|c|c|c|c|c|c|c|}
\hline \multirow{3}{*}{$\begin{array}{l}\text { Wind speed } \\
\text { direction } \phi\end{array}$} & \multicolumn{12}{|c|}{ Partitions } \\
\hline & \multicolumn{2}{|c|}{$\mathcal{P}_{1}$} & \multicolumn{2}{|c|}{$\mathcal{P}_{2}$} & \multicolumn{2}{|c|}{$\mathcal{P}_{3}$} & \multicolumn{2}{|c|}{$\mathcal{P}_{4}$} & \multicolumn{2}{|c|}{$\mathcal{P}_{5}$} & \multicolumn{2}{|c|}{$\mathcal{P}_{6}$} \\
\hline & $n_{1}$ & $\mu_{1}(\mathrm{~s})$ & $n_{2}$ & $\mu_{2}(\mathrm{~s})$ & $n_{3}$ & $\mu_{3}(\mathrm{~s})$ & $n_{4}$ & $\mu_{4}(\mathrm{~s})$ & $n_{5}$ & $\mu_{5}(\mathrm{~s})$ & $n_{6}$ & $\mu_{6}(\mathrm{~s})$ \\
\hline $0^{\circ}$ & 7 & 0.28 & 7 & 0.28 & 7 & 0.28 & 7 & 0.28 & 7 & 0.28 & 7 & 0.28 \\
\hline $30^{\circ}$ & 9 & 0.30 & 8 & 0.28 & 8 & 0.26 & 5 & 0.21 & 5 & 0.18 & 7 & 0.25 \\
\hline $60^{\circ}$ & 12 & 0.16 & 6 & 0.15 & 6 & 0.15 & 6 & 0.15 & 6 & 0.15 & 6 & 0.15 \\
\hline $90^{\circ}$ & 9 & 0.3 & 9 & 0.3 & 6 & 0.18 & 6 & 0.18 & 6 & 0.18 & 6 & 0.18 \\
\hline
\end{tabular}

number of subsets required for finding the optimal partition. For the chosen parameters, the minimum number of subsets ensuring the desired values of $\sigma^{*}$ and $\beta^{*}$ is $m=6$, i.e. $\mathcal{K}=\{1, \ldots, 6\}$. Thus, the maximum resource-feeding index for each subset $\tau_{l}^{*}$, with $l \in \mathcal{K}$, along with the corresponding turbine $i \in \mathcal{V}_{l}$ for which $\tau_{i}=\tau_{l}^{*}$ is: $\tau_{1}^{*}=3$ for $\mathrm{T}_{(42,35)}, \tau_{2}^{*}=3$ for $\mathrm{T}_{(27)}, \tau_{3}^{*}=2$ for $\mathrm{T}_{(41,42)}, \tau_{4}^{*}=2$ for $\mathrm{T}_{38}, \tau_{5}^{*}=2$ for $\mathrm{T}_{(13,14)}$ and $\tau_{6}^{*}=3$ for $\mathrm{T}_{(21,28)}$.

The weights in (5a) were set as $w_{1}=0.7, w_{2}=0.1$ and $w_{3}=0.2$, in order to provide the highest priority at the maximization of the coupling due to the wake effect among turbines in the same subsets. Meanwhile, lower priority is set for the third objective function $f_{3}$, to balance the number of turbines at each partition by minimizing the difference of the number of turbines belonging to different partitions. Finally, the lower priority is set for the second objective $f_{2}$ since it is only relevant to ensure an optimal solution at partitioning problem when there is no wake interaction among the turbines. It is worthwhile to note that increasing the value of $w_{3}$ at the expense of the weight $w_{1}$ might yield a different result since the difference among the numbers of turbines in the subset gets close to zero.

\section{Control design}

In order to design the MPC strategies, the time constant in (11) was set to $\mu=0.125 \mathrm{~s}$. Notice that this value has been obtained by modeling the wind turbine power input/power output response as a first-order system. For the PLC, the sampling time used to discretize the partition model in (11) was set to $0.05 \mathrm{~s}$, the prediction horizon to $H_{\mathrm{p}}=3$, and the weights in (13a) to $\gamma_{1}=0.45, \gamma_{2}=0.35$ and $\gamma_{3}=0.2$. Thus, total power regulation and maximization of available power have higher priority than control input limitations. For the CC in (14), the sampling time was set to $0.1 \mathrm{~s}$, the prediction horizon at $H_{\mathrm{p}}=3$, and the weights to $Q_{\xi}=0.8$ and $\mathbf{S}=0.2 \cdot \mathbf{I}_{6}$ to prioritize the tracking of the power demanded by the grid. The time constants $\mu_{l}$ for each partition $l \in \mathcal{K}$ are given in Table 2 . They have been computed by modeling the open-loop power response for a given power input of each partition as a first-order system with time constant $\mu_{i}$. Notice that such an assumption can be done when the internal variables (individual power, mechanical load, pitch reference, etc.) are not in the controller design [12]. As the number of turbines in each subsets is different, the time constants $\mu_{l}$ needed to approximate the dynamics of each subset are also different.

In order to highlight the effect of redistributing the power contribution of each wind turbine in the partition, in all simulations, for all $t<t_{0}=50$ s the weight on the control input in (13) is selected as $\mathbf{R}=\mathbf{I}_{n_{l}}$, i.e., equal contribution is required for every turbine, whereas, for all $t \geq t_{0}$ the contribution are distributed as discussed in Section 4.

\subsection{Test 1: Power regulation}

First, the proposed control strategy was evaluated in the case of a predominant wind speed direction of $\phi=30^{\circ}$ and a power demanded by the TSO of $P_{\mathrm{dem}}=60 \mathrm{MW}$. Hence, the wind farm operates in derated mode, as the total available power simulated in steady-state conditions $P_{\mathrm{av}, \text { to }}$ is almost $60 \%$ higher than the power demand. The 
partitioning in this case results in the following subsets:

$$
\begin{aligned}
& \mathcal{P}_{1}=\{8,16,17,24,25,33,34,35,42\}, \\
& \mathcal{P}_{2}=\{1,2,9,10,18,19,26,27\}, \\
& \mathcal{P}_{3}=\{15,22,23,31,32,39,40,41\}, \\
& \mathcal{P}_{4}=\{29,30,36,37,38\}, \\
& \mathcal{P}_{5}=\{5,6,7,13,14\}, \\
& \mathcal{P}_{6}=\{3,4,11,12,20,21,28\} .
\end{aligned}
$$

Figure 4 illustrates the partitioning and the wake effect.

Figure 5a shows the total power generated $P_{\mathrm{w}, \text { tot }}$ (black line), the set-point $P_{\text {dem }}$ (gray line) and the total available power $P_{\mathrm{av}, \text { tot }}$ (blue line). The redistribution of the power contribution starts to affect the available power only after $t=t_{0}+t_{\mathrm{w}}$, where $t_{\mathrm{w}} \approx 60 \mathrm{~s}$ is the time the wind takes to travel from one turbine to the next downstream one [31]. Comparing the initial and final values of $P_{\text {av,tot }}$, it can be observed that the power contributions imposed by each PLC are capable of increasing the power reserve with about $2.7 \%$ (from $167.5 \mathrm{MW}$ at $t=t_{0}$ to $172 \mathrm{MW}$ at $t=600 \mathrm{~s}$ ) without affecting the power demand tracking.

Figure $5 \mathrm{~b}$ and $5 \mathrm{c}$ show the available and generated powers, $P_{\mathrm{av}, l}^{\mathrm{p}}$ and $P_{\mathrm{w}, l}^{\mathrm{p}}$ respectively, for each subset. It can be observed that Subsets $\mathcal{P}_{4}$ and $\mathcal{P}_{5}$ make the higher contributions $\left(P_{\mathrm{w}, 5}^{\mathrm{p}}=12.4 \mathrm{MW}, P_{\mathrm{w}, 4}^{\mathrm{p}}=11 \mathrm{MW}\right)$, whereas the lowest ones are observed in case of Subsets $\mathcal{P}_{1}$ and $\mathcal{P}_{2}\left(P_{\mathrm{w}, 1}^{\mathrm{p}}=8.2 \mathrm{MW}, P_{\mathrm{w}, 2}^{\mathrm{p}}=8.8 \mathrm{MW}\right)$. The remaining subsets produce $P_{\mathrm{w}, 3}^{\mathrm{p}}=P_{\mathrm{w}, 6}^{\mathrm{p}}=9.6 \mathrm{MW}$. In Figure $5 \mathrm{~b}$, the available power $P_{\mathrm{av}, l}^{\mathrm{p}}$ increases in all subsets compared with the initial values, except for Subsets $\mathcal{P}_{3}$ showing a small reduction (close to $1.5 \%$ ).

Figure 6 shows the power generated by each turbine in each partition. It can be observed the backward distribution of the power contribution of each turbine imposed by the PLCs in each partition. The largest contribution is done by the most downstream turbines whereas the most upstream ones tend to reduce the power generation. In this scheme, the power generation of some of the upstream turbines reaches the minimum value $P_{\min }$.

Figure 7 shows the total available power for different values of power demand: $P_{\mathrm{dem}}=60 \mathrm{MW}$ (blue line), $70 \mathrm{MW}$ (red line), $80 \mathrm{MW}$ (yellow line) and 100MW (purple line). It can be observed that, in cases of high derating operations $\left(P_{\mathrm{dem}}<80 \mathrm{MW}\right)$, the total available power increases compared to the values obtained with the uniform power contribution scheme $\left(t<t_{0}\right)$. In these circumstances, the coefficient $\lambda$ used in the weight $\mathbf{R}$ in (13a) results to be 0.64 and 0.5 , respectively, and the power contribution of each turbine at each subset is determined according to the backward distribution. On the other hand, when the power demand is close to $P_{\mathrm{av}, \text { tot }}, \lambda$ is close to zero and the matrices $\mathbf{R}$ tend to $\mathbf{I}_{n_{l}}$. As a result, the set-points $P_{\mathrm{r}, i}$ are similar and every turbine in the partition is required to contribute approximately the same power level. The motivation for using this scheme is due to the fact that when $P_{\mathrm{dem}}$ is high, turbines reach their maximum power limits, especially the most downstream ones, and the backward distribution stops being effective.

\subsection{Test 2: Sensitivity to wind speed directions}

In this test, the proposed non-centralized control strategy is evaluated when the partitioning does not correspond to the exact predominant wind speed direction. In this test, the proposed non-centralized control strategy is evaluated when the partitioning does not correspond to the exact predominant wind speed direction. As previously mentioned, the subset is computed offline for different sets of directions and selected from a table with an estimated wind speed direction. The aim of this test is to analyze the effect of this approximation on the robustness and performance of the proposed control scheme when there is uncertainty in the measures of wind speed directions.

Figure 8 presents both the total available and the total generated power for the actual predominant wind directions $\phi_{\text {real }} \in\left\{15^{\circ}, 20^{\circ}, 30^{\circ}, 40^{\circ}, 50^{\circ}\right\}$ while the MPC controller is designed for $\phi=30^{\circ}$. It can be observed that in all cases, the controller is able to deliver the power demanded by the TSO, $P_{\mathrm{dem}}=60 \mathrm{MW}$. Moreover, the backward power distribution is also capable of increasing the total available power. Nevertheless, when the direction $\phi$ used for the design does not correspond to the real predominant wind speed direction $\phi_{\text {real }}$, some deterioration in the system response can be observed. The available power profiles in Figure $8 \mathrm{~b}$ indicate that the largest increase regarding the initial values $\left(t<t_{0}\right)$, when the backward distribution is not active, is observed in the case where the $\phi$ used in the 

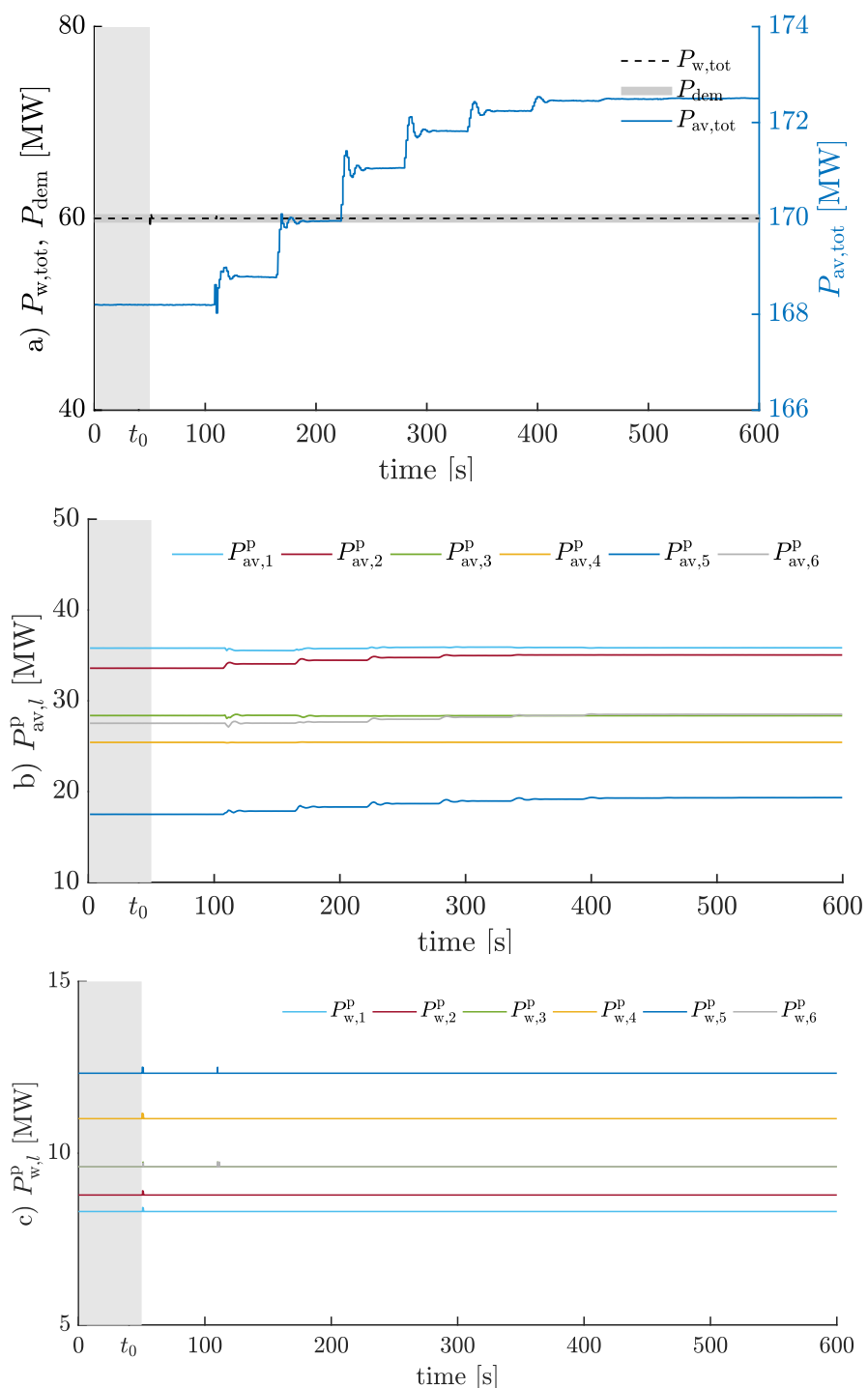

Figure 5: Test 1: Closed-loop response for $P_{\mathrm{dem}}=60 \mathrm{MW}, \phi=30^{\circ}$, and $v_{\infty}=11 \mathrm{~m} / \mathrm{s}$. a) Total generated and available power, b) Available power for each partition, c) Generated power for each partition. 

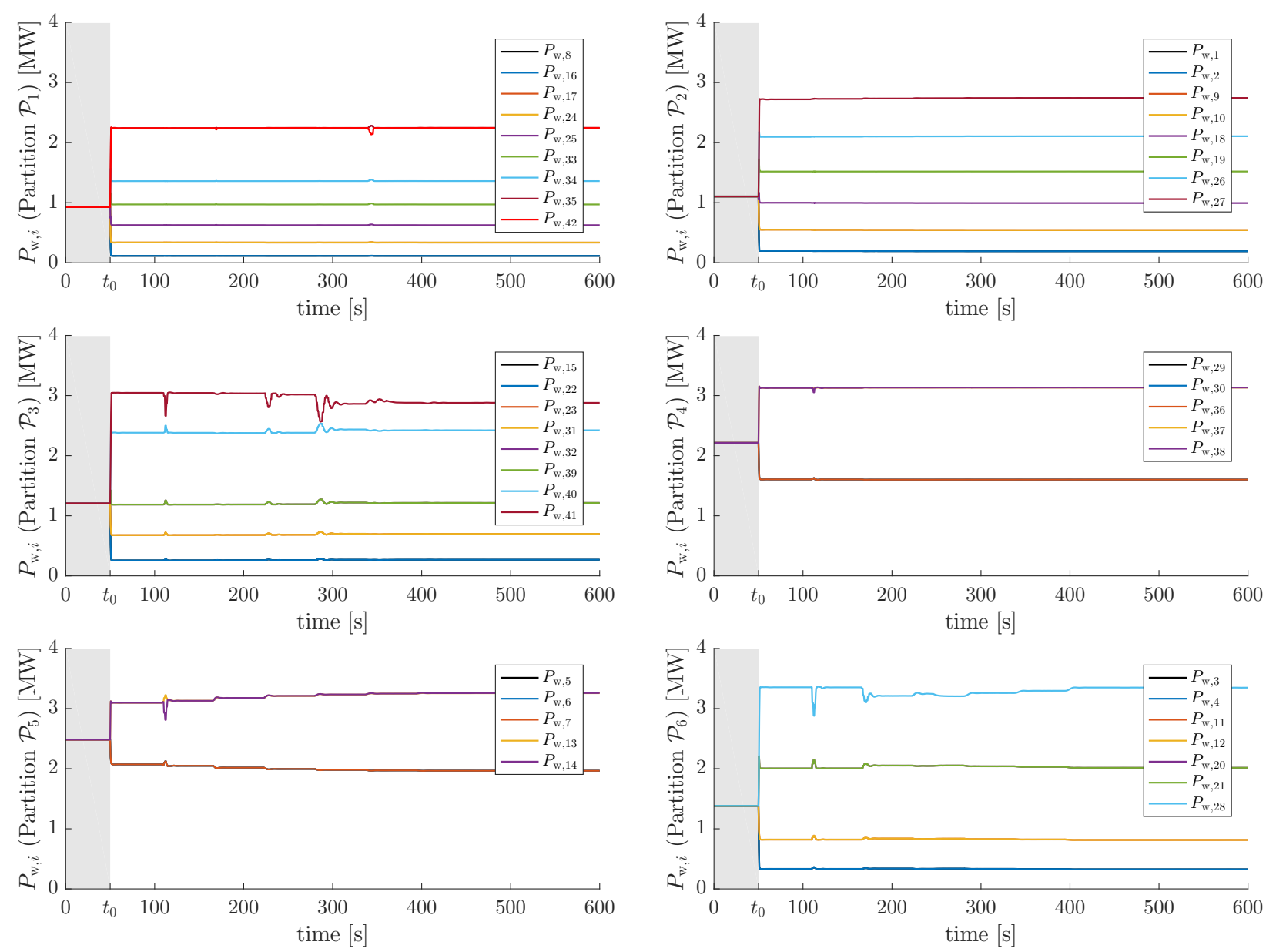

Figure 6: Test 1: Closed loop response for $P_{\mathrm{dem}}=60 \mathrm{MW}, \phi=30^{\circ}$, and $v_{\infty}=11 \mathrm{~m} / \mathrm{s}$. Generated power by every turbine in each partition.

controller design coincides with $\phi_{\text {real }}$. Notice that the initial values are different for each $\phi_{\text {real }}$ as wind speed deficit depends on this angle. The increase in the available power can be compared using the expression

$$
\Delta P_{\mathrm{av}}=\frac{P_{\mathrm{av}, \mathrm{tot}}(t=600 \mathrm{~s})-P_{\mathrm{av}, \mathrm{tot}}\left(t_{0}\right)}{P_{\mathrm{av}, \mathrm{tot}}\left(t_{0}\right)} \cdot 100 \%,
$$

with $P_{\text {av,tot }}(t=600 \mathrm{~s})$ and $P_{\text {av,tot }}\left(t_{0}\right)$ respectively the available power at the steady-state when the backward distribution is followed and the available power when the turbines provide the same power. Figure $8 \mathrm{c}$ shows the values of $\Delta P_{\text {av }}$ corresponding to the aforementioned cases.

The previous analysis can be repeated to cover the entire $360^{\circ}$ range and to propose a set of sectors in which a controller designed for a given direction will work properly also for different wind direction belonging to the same sector. This approximation avoids the problem of changing the controllers for any change of wind speed direction and provides a more robust control strategy. Table 3 lists the directions used to compute the subsets and the sectors in which the corresponding controllers are valid. Here, the entire $360^{\circ}$ range was divided in equal sectors. If a wind speed history of the farm site is available, the identification of the sectors in Table 3 can be defined using the corresponding wind rose information. In fact, if the specific distribution of the wind speed direction is known, then the partitions (and the size of each angle sector) can be updated according to the frequency of the wind direction. 


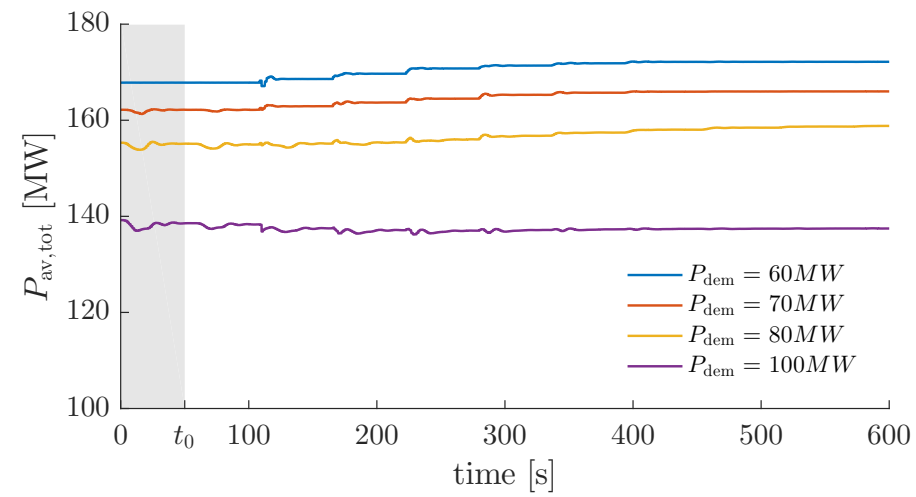

Figure 7: Test 1: Total available power for several set-points of $P_{\text {dem }}$.
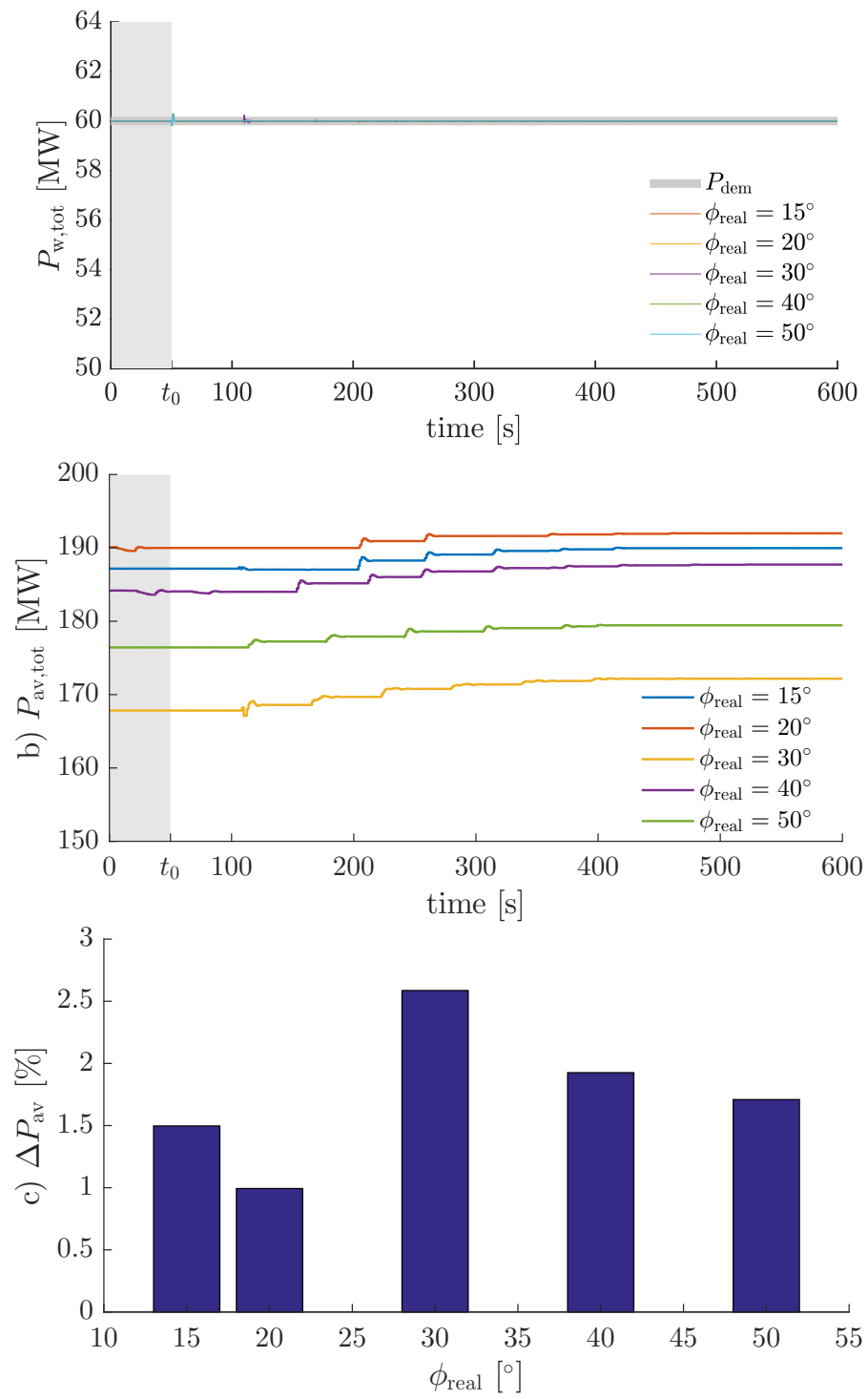

Figure 8: Test 2: Closed-loop response when the controller is based on a wind speed direction of $30^{\circ}$ but the real direction is $\phi_{\text {real }}$. a) Total generated power, b) Total available power. c) Total available power increment (16). 
Table 3: Test 2: Angle $\phi$ used in partitioning and the sectors in which the controller performs properly.

\begin{tabular}{cc}
\hline Design angle $\phi\left[^{\circ}\right]$ & Validity sector $\left[{ }^{\circ}\right]$ \\
\hline 0 & $-5 \leq \phi<25$ \\
30 & $25 \leq \phi<55$ \\
60 & $55 \leq \phi<85$ \\
90 & $85 \leq \phi<115$ \\
120 & $115 \leq \phi<145$ \\
150 & $145 \leq \phi<175$ \\
180 & $175 \leq \phi<205$ \\
210 & $205 \leq \phi<235$ \\
240 & $235 \leq \phi<265$ \\
270 & $265 \leq \phi<295$ \\
300 & $295 \leq \phi<325$ \\
330 & $325 \leq \phi<355$ \\
\hline
\end{tabular}

\subsection{Test 3: Comparison between non-centralized and centralized control approaches}

One of the aims of proposing a non-centralized control approach is to reduce the computation time. In order to evaluate this point, in this section, the proposed scheme is compared with a centralized control scheme. The simulations were performed for a predominant wind speed direction of $30^{\circ}$ and for three different partition schemes: $m=4,5,6$. The centralized control scheme corresponds to the PLC with one partition. The total generated and the total available power for all cases can be seen in Figure 9. Clearly, the best performance is achieved with the centralized scheme, which has more information but also requires more computation time. Nevertheless, it can be observed that the non-centralized schemes are able to achieve values of total available power close to the centralized option. The increase in the available power $\Delta P_{\text {av }}$ (equation (16)) results in $0.6 \%$ for the case $m=4$ (blue line) and in $2.6 \%$ for the case $m=6$ (yellow line), which is close to the centralized values.

With the aim of comparison, Table 4 lists computation times, total available power and the increase in the power reserve. The computation times were determined using a computer with an Intel i7 processor, 8 GB of RAM running Windows 10. The first and second rows in Table 4 present the time the solver needs to find the solution of the optimization problems (10) and (14). Notice that in the non-centralized cases, the solver times for local controllers correspond to the worst case (partitions with the highest number of turbines). As the CC and each PLC run in parallel (on different computers), the estimation of the total solver time must be taken as the largest time value, which is indicated as $t_{s, \text { tot }}=\max \left(\max \left(t_{s}(\mathrm{PLC})_{i}\right), t_{s}(\mathrm{CC})\right)$, with $i \in \mathcal{K}$ and $t_{s}(\mathrm{CC})$ the solver time for the central controller. The computation improvement between the centralized case and the case of 6 partitions can be evaluated as

$$
\Delta t_{s}=\frac{t_{s}(\mathrm{CMPC})-t_{s, \mathrm{tot}}}{t_{s}(\mathrm{CMPC})} \cdot 100 \% .
$$

Therefore, the proposed scheme achieves an improvement of almost $70 \%$ of the computation time, with a similar response for the total available power.

On the other hand, Table 5 compares the improvement in the available power $\Delta P_{\text {av,tot }}$ obtained with the centralized and no-centralized schemes for wind speed direction $\phi \in\{0,20,40,50\}$ when the subset corresponding to the sector $25 \leq \phi \leq 55$ is used. It can be observed that in all cases, the no-centralized approach reaches values close to the centralized ones.

\section{Conclusions}

This paper has presented a novel active power control strategy for wind farms based on non-centralized predictive control with a wake-based partitioning approach. Such novel control strategy aims to reduce the high communication and computational burden that can be an issue for guaranteeing online control of large wind farms. To this end, a 

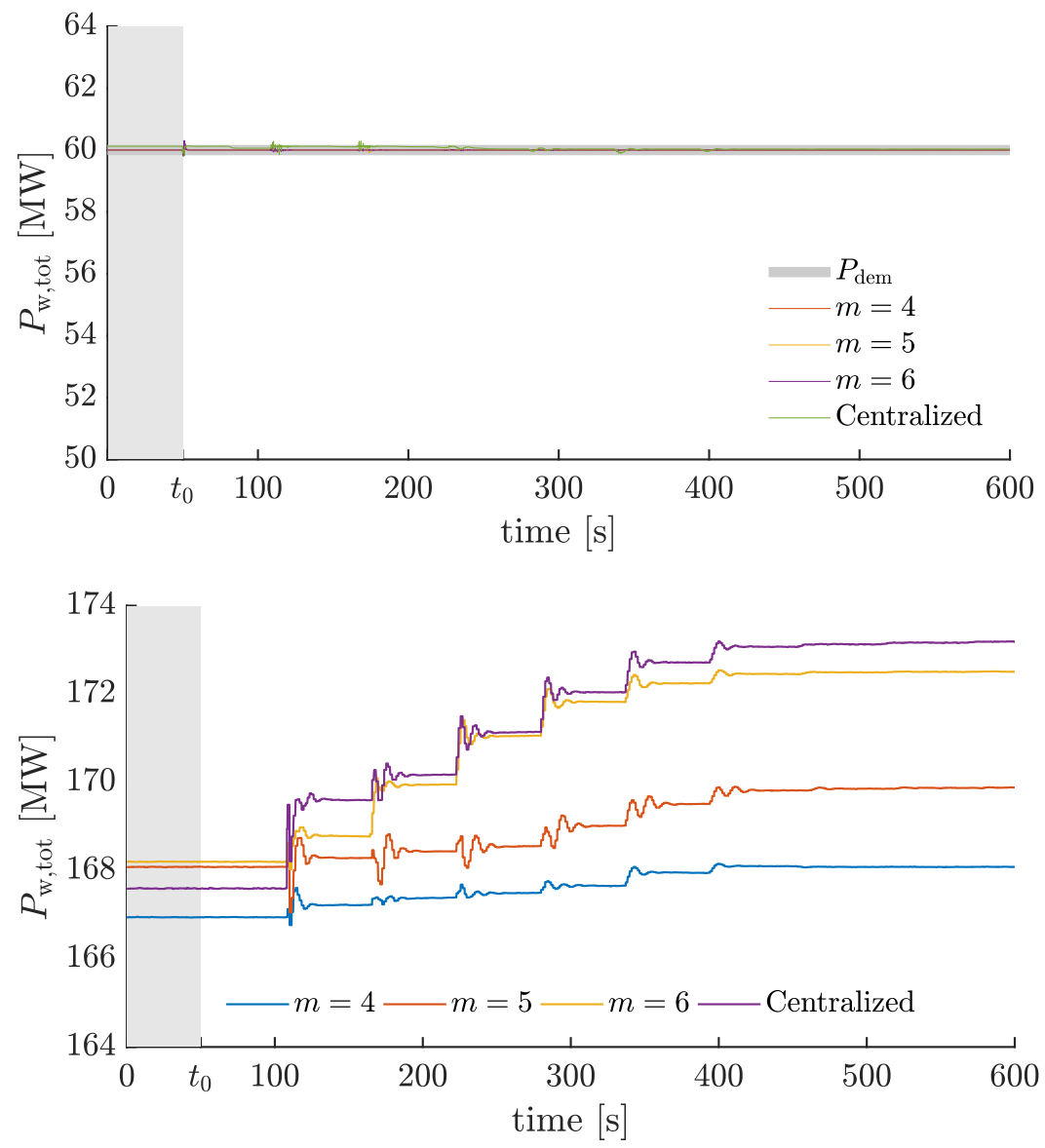

Figure 9: Test 3: Comparison among non-centralized schemes with several numbers of partitions $m$ and a centralized approach. a) Total generated power. b) Total available power.

Table 4: Solver time for the centralized and non-centralized MPC approaches (PLC and CC) together with the percentage of improvement with respect to the centralized case and total available power at steady state.

\begin{tabular}{llllll}
\hline & \multicolumn{3}{c}{ Centralized (CMPC) } & \multicolumn{4}{c}{ Decentralized } \\
\cline { 2 - 6 } & & $\mathrm{m}$ & 4 & 5 & 6 \\
\hline Solver time [s] & 0.1081 & $t_{s}(\mathrm{PLC}):$ & 0.009 & 0.0079 & 0.0046 \\
& & $t_{s}(\mathrm{CC}):$ & 0.0898 & 0.0401 & 0.0328 \\
\hline Computational improvement $\left(\Delta t_{s}\right)[\%]$ & 0 & & 18 & 63 & 70 \\
\hline$\Delta P_{\text {av, tot }}[\%]$ & 3.06 & & 0.68 & 1.07 & 2.59 \\
\hline
\end{tabular}

Table 5: Percentage of improvement of total available power at steady state with respect to the centralized case

\begin{tabular}{cccccc}
\hline & \multicolumn{4}{c}{ Wind speed direction $\left[^{\circ}\right]$} \\
\cline { 3 - 6 } & & 0 & 20 & 40 & 50 \\
\hline \multirow{2}{*}{$\Delta P_{\text {av,tot }}[\%]$} & Centralized & 1.90 & 1.91 & 2.98 & 1.88 \\
& Decentralized & 1.47 & 1.32 & 2.24 & 1.43 \\
\hline
\end{tabular}


partitioning algorithm, stated as a mixed-integer multi-objective problem, has been designed to divide the wind farm into smaller subsets of turbines such that the amount of information shared with the central control could be reduced and the overall system resiliency is improved. The subsets are identified such that the couplings due to the wake effects among the turbines within different partitions are minimized. Therefore, each partition is considered as an independent unit controlled by a local predictive controller defined to solve a multi-objective optimal control problem in order to provide the power profile set by the central controller and optimally regulate the power set-points among the turbines such that the overall available power is improved.

The results show that the non-centralized approach reduces consistently the computational costs with respect to a fully centralized strategy, allowing the proposed controller to be suitable for real-time applications. Moreover, the drawback due to the reduction of the information sharing only slightly affects the overall performance of the wind farm generation. In fact, the results show that the decreasing of the available power is lower than $1 \%$ with respect to the centralized case and, increasing the number of partitions, the available power converge to the centralized case.

\section{Acknowledgments}

This work has received funding from the European Union's Horizon 2020 research and innovation programme under the Marie Skłodowska-Curie grant agreement No 675318 (INCITE).

\section{References}

[1] G. W. E. Council, Global wind report (2018).

[2] ENTSO-E, Annual report (Apr. 2017).

[3] J. Aho, A. Buckspan, J. Laks, P. Fleming, Y. Jeong, F. Dunne, M. Churchfield, L. Pao, K. Johnson, A tutorial of wind turbine control for supporting grid frequency through active power control, in: American Control Conference (ACC), 2012, 2012 , pp. 3120-3131.

[4] M. Tsili, S. Papathanassiou, A review of grid code technical requirements for wind farms, IET Renewable Power Generation 3 (3) (2009) 308-332.

[5] A. D. Hansen, P. Sørensen, F. Iov, F. Blaabjerg, Centralised power control of wind farm with doubly fed induction generators, Renewable Energy 31 (7) (2006) 935-951.

[6] W. Qiuwei, S. Yuanzhang, Modeling and Modern Control of Wind Power, John Wiley \& Sons, 2018.

[7] J. Barreiro-Gomez, C. Ocampo-Martinez, N. Quijano, Time-varying partitioning for predictive control design: Density-games approach, Journal of Process Control 75 (2019) $1-14$.

[8] C. Ocampo-Martinez, S. Bovo, V. Puig, Partitioning approach oriented to the decentralised predictive control of large-scale systems, Journal of Process Control 21 (5) (2011) 775-786.

[9] J. M. Grosso, C. Ocampo-Martinez, V. Puig, A distributed predictive control approach for periodic flow-based networks: application to drinking water systems, International Journal of Systems Science 48 (14) (2017) 3106-3117.

[10] J. Annoni, C. Bay, K. Johnson, E. Dall'Anese, E. Quon, T. Kemper, P. Fleming, A framework for autonomous wind farms: Wind direction consensus, Wind Energy Science Discussions 2018 (2018) 1-17.

[11] N. Gionfra, G. Sandou, H. Siguerdidjane, D. Faille, P. Loevenbruck, Wind farm distributed PSO-based control for constrained power generation maximization, Renewable Energy 133 (2019) 103-117.

[12] V. Spudic, M. Baotic, Fast coordinated model predictive control of large-scale distributed systems with single coupling constraint, in: European Control Conference (ECC),, 2013, pp. 2783-2788.

[13] H. Zhao, Q. Wu, Q. Guo, H. Sun, Y. Xue, Distributed model predictive control of a wind farm for optimal active power control part II: Implementation with clustering-based piece-wise affine wind turbine model, IEEE Transactions on Sustainable Energy 6 (3) (2015) $840-849$.

[14] J. Annoni, C. Bay, T. Taylor, L. Pao, P. Fleming, K. Johnson, Efficient optimization of large wind farms for real-time control, in: Proc. of the American Control Conference (ACC), 2018, pp. 6200-6205.

[15] C. J. Bay, J. Annoni, T. Taylor, L. Pao, K. Johnson, Active power control for wind farms using distributed model predictive control and nearest neighbor communication, in: Proc. of the American Control Conference (ACC), 2018, pp. 682-687.

[16] S. Baros, M. D. Ilić, Distributed torque control of deloaded wind DFIGs for wind farm power output regulation, IEEE Transactions on Power Systems 32 (6) (2017) 4590-4599.

[17] X. Gao, K. Meng, Z. Y. Dong, D. Wang, M. S. El Moursi, K. P. Wong, Cooperation-driven distributed control scheme for large-scale wind farm active power regulation, IEEE Transactions on Energy Conversion 32 (3) (2017) 1240-1250.

[18] B. Zhang, M. Soltani, W. Hu, P. Hou, Q. Huang, Z. Chen, Optimized power dispatch in wind farms for power maximizing considering fatigue loads, IEEE Transactions on Sustainable Energy 9 (2) (2017) 862-871.

[19] H. Zhao, Q. Wu, Q. Guo, H. Sun, Y. Xue, Optimal active power control of a wind farm equipped with energy storage system based on distributed model predictive control, IET Generation, Transmission \& Distribution 10 (3) (2016) 669-677.

[20] S. Siniscalchi-Minna, C. Ocampo-Martinez, F. D. Bianchi, M. De Prada-Gil, B. De Schutter, Partitioning approach for large wind farms: Active power control for optimizing power reserve, in: Proc. of the Conference on Decision and Control (CDC), 2018, pp. 3183-3188.

[21] J. D. Grunnet, M. Soltani, T. Knudsen, M. N. Kragelund, T. Bak, Aeolus toolbox for dynamics wind farm model, simulation and control, in: Proc. of the European Wind Energy Conference \& Exhibition, EWEC, 2010.

[22] F. D. Bianchi, H. De Battista, R. J. Mantz, Wind turbine control systems: principles, modelling and gain scheduling design, Springer, 2006. 
[23] I. Katic, Hojstrup, N. O. Jensen, A simple model for cluster efficiency, in: Proc. of the European Wind Energy Association Conference and Exhibition, 1986, pp. 407-410.

[24] R. J. Barthelmie, K. Hansen, S. T. Frandsen, O. Rathmann, Schepers, Modelling and measuring flow and wind turbine wakes in large wind farms offshore, Wind Energy 12 (5) (2009) 431-444.

[25] V. Gevorgian, Y. Zhang, E. Ela, Investigating the impacts of wind generation participation in interconnection frequency response, IEEE Transactions on Sustainable Energy 6 (3) (2015) 1004-1012.

[26] J. Barreiro-Gomez, Partitioning for large-scale systems: Sequential DMPC design, in: The Role of Population Games in the Design of Optimization-Based Controllers, Springer, 2019, pp. 163-178.

[27] A. Bemporad, M. Morari, Control of systems integrating logic, dynamics, and constraints, Automatica 35 (3) (1999) $407-427$.

[28] S. Siniscalchi-Minna, F. D. Bianchi, M. De Prada-Gil, C. Ocampo-Martinez, A wind farm control strategy for power reserve maximization, Renewable Energy 131 (2019) 37-44.

[29] J. Jonkman, S. Butterfield, W. Musial, G. Scott, Definition of a 5-mw reference wind turbine for offshore system development, National Renewable Energy Laboratory, Golden, CO, Technical Report No. NREL/TP-500-38060 (2009).

[30] J. Lofberg, Yalmip: A toolbox for modeling and optimization in MATLAB, in: Proc. of the IEEE International Symposium on Computer Aided Control Systems Design, 2005, pp. 284-289.

[31] U. Ciri, M. A. Rotea, S. Leonardi, Model-free control of wind farms: A comparative study between individual and coordinated extremum seeking, Renewable Energy 113 (2017) 1033-1045. 